\title{
Recent Progress in General Sparse Direct Solvers
}

\author{
Anshul Gupta \\ IBM T.J. Watson Research Center \\ P.O. Box 218, Yorktown Heights, NY 10598 \\ anshul@watson.ibm.com \\ http://www.cs.umn.edu/ agupta/wsmp.html
}

\begin{abstract}
During the past few years, algorithmic improvements alone have shaved almost an order of magnitude off the time required for the direct solution of general sparse systems of linear equations. Combined with a similar increase in the performance to cost ratio due to hardware advances during this period, current sparse solver technology makes it possible to solve those problems quickly and easily that might have been considered impractically large until recently. In this paper, we compare the performance of some commonly used software packages for solving general sparse systems. In particular, we demonstrate the consistently high level of performance achieved by WSMP - the most recent of such solvers. We compare the various algorithmic components of these solvers and show that the choices made in WSMP enable it to run two to three times faster than the best amongst other similar solvers. As a result, WSMP can factor some of the largest sparse matrices available from real applications in a few seconds on 4-CPU workstation.
\end{abstract}

\section{Introduction}

Developing an efficient parallel, or even serial, direct solver for general sparse systems of linear equations is a challenging task that has been the subject of research for the past four decades. Several breakthroughs have been made during this time. As a result, a number of very competent software packages for solving such systems are available $[2,468,17 / 23,2625]$.

In this paper, we compare the performance of some commonly used software packages for solving general sparse systems and show that during the past few years, algorithmic improvements alone have shaved an order of magnitude off the time required to factor general sparse matrices. Combined with a similar increase in the performance to cost ratio due to hardware advances during this period, current sparse solver technology makes it possible to solve those problems quickly and easily that might have been considered impractically large until recently. We demonstrate the consistently high level of performance achieved by the Watson Sparse Matrix Package (WSMP) and show that it can factor some of the largest sparse matrices available from real applications in a few seconds on 4-CPU workstation. The WSMP project's original aim was to develop a scalable

V.N. Alexandrov et al. (Eds.): ICCS 2001, LNCS 2073, pp. 823 8312001.

(C) Springer-Verlag Berlin Heidelberg 2001 
parallel general sparse solver for a distributed-memory parallel computer like the IBM SP. However, after completing the serial version of the solver, we realized that we couldn't find enough large problems that would justify the use of an SP with several nodes. Therefore, we tailored the parallel version to use a few CPU's in a shared-memory environment. It is one of the objectives of this paper to make the user community aware of the robustness and speed of the current sparse direct solver technology and encourage scientists and engineers to develop bigger models with larger sparse systems so that the full potential of these solvers can be utilized.

\section{Comparison of Serial Performance of Some General Sparse Solvers}

In this section, we compare the performance of some of the most well-known software packages for solving sparse systems of linear equations on a single CPU of an IBM RS6000 S80. This is a $600 \mathrm{Mhz}$ processor with a $64 \mathrm{~KB}$ level-1 cache and a peak theoretical speed of of 1.2 Gigaflops. 2 GB of memory was available to each solver. A more detailed comparison of the serial and the parallel versions of these solvers can be found in [19. Table 1 lists all the test matrices, their dimensions, number of nonzeros, and application areas of their origin.

Table 2 lists the serial LU factorization time taken by UMFPACK [8], SuperLU [9], SPOOLES 4, SuperLU dist 22 23], MUMPS [1!2], and WSMP [15 17]. This table also lists the year in which the latest version of each of these packages became available. The best factorization time for each matrix using any solver released before year 2000 is shown in italics and the overall best factorization time is shown in boldface.

The most striking observation in Table 2 is the range of times that different packages available before 1999 would take to factor the same matrix. It is not uncommon to notice the fastest solver being faster than the slowest one by one to two orders of magnitude. Additionally, none of them gave a consistent level of performance. For example, UMFPACK is 13 times faster than SPOOLES on e40r5000 but 14 times slower on fidap011. MUMPS is clearly the fastest and the most robust amongst the solvers available before 2000. However, the latest solver WSMP appears to be about two and half times faster than MUMPS on this machine on an average. WSMP also has the most consistent performance. It has the smallest factorization time for all but two matrices and is the only solver that does not fail on any of the test matrices.

\section{Algorithmic Features of the Solvers}

In this section, we list the algorithms and strategies that these solvers use for the symbolic and numerical phases of the computation of the LU factors of a general sparse matrix. We then briefly discuss the role of these choices on the performance of the solvers. The description of all these algorithms is beyond 
Table 1. Test matrices with their order (N), number of nonzeros (NNZ), and the application area of origin.

\begin{tabular}{|l|r|r|c|}
\hline Matrix & \multicolumn{1}{|c|}{$N$} & \multicolumn{1}{|c|}{$N$ $Z$} & Application \\
\hline af23560 & 23560 & 484256 & Fluid dynamics \\
av41092 & 41092 & 1683902 & F.E.M. \\
bayer01 & 57735 & 277774 & Chemistry \\
bbmat & 38744 & 1771722 & Fluid dynamics \\
comp2c & 16783 & 578665 & Linear programming \\
e40r0000 & 17281 & 553956 & Fluid dynamics \\
e40r5000 & 17281 & 553956 & Fluid dynamics \\
ecl32 & 51993 & 380415 & Electrical eng. \\
epb3 & 84617 & 463625 & Thermodynamics \\
fidap011 & 16614 & 1091362 & F.E.M. \\
fidapm11 & 22294 & 623554 & F.E.M. \\
invextr1 & 30412 & 1793881 & Fluid dynamics \\
lhr34c & 35152 & 764014 & Chemical eng. \\
lhr71c & 70304 & 1528092 & Chemical eng. \\
mil053 & 530238 & 3715330 & F.E.M. \\
mixtank & 29957 & 1995041 & Fluid dynamics \\
nasasrb & 54870 & 2677324 & F.E.M. \\
onetone1 & 36057 & 341088 & Circuit simulation \\
onetone2 & 36057 & 227628 & Circuit simulation \\
pre2 & 659033 & 5959282 & Circuit simulation \\
raefsky3 & 21200 & 1488768 & Fluid dynamics \\
raefsky4 & 19779 & 1316789 & Fluid dynamics \\
rma10 & 46835 & 2374001 & Fluid dynamics \\
tib & 18510 & 145149 & Circuit simulation \\
twotone & 120750 & 1224224 & Circuit simulation \\
venkat50 & 62424 & 1717792 & Fluid dynamics \\
wang3 & 26064 & 177168 & Circuit simulation \\
wang4 & 26068 & 177196 & Circuit simulation \\
\hline
\end{tabular}

the scope of this paper. The reader should be able to find them in the citations provided.

\section{UMFPACK 8}

- Fill reducing ordering: Approximate minimum degree [7] on unsymmetric structure, combined with suitable numerical pivot search during LU factorization.

- Task dependency graph: Directed acyclic graph.

- Numerical factorization: Unsymmetric-pattern multifrontal.

- Pivoting strategy: Threshold pivoting implemented by row-exchanges.

2. SuperLU [9]

- Fill reducing ordering: Multiple minimum degree (MMD) 13 on the symmetric structure of $A A^{T}$ or $A+A^{T}$, where $A$ is the original coefficient matrix. 
Table 2. LU Factorization time on a single CPU (in seconds) for UMFPACK, SuperLU, SPOOLES, SuperLU ${ }_{d i s t}$, MUMPS, and WSMP, respectively. The best pre-2000 time is shown in italics and the overall best time is shown in boldface.

\begin{tabular}{||l||c|c|c|c|c|c||}
\hline \hline Year $\rightarrow$ & 1994 & 1997 & 1998 & 1999 & 1999 & 2000 \\
\hline Matrix & UMFPACK & SuperLU & SPOOLES & SuperLU dist & MUMPS & WSMP \\
\hline \hline af23560 & 45.5 & 31.9 & 10.5 & 14.7 & 8.93 & $\mathbf{6 . 1 9}$ \\
av41092 & 186. & 772. & Fail & Fail & 30.6 & $\mathbf{8 . 4 7}$ \\
bayer01 & 1.76 & 2.40 & Fail & 3.23 & 2.26 & $\mathbf{1 . 3 3}$ \\
bbmat & 682. & 214. & 97.7 & Fail & 113. & $\mathbf{3 6 . 7}$ \\
comp2c & 120. & 3403 & 287. & 42.0 & 29.3 & $\mathbf{4 . 0 8}$ \\
e40r5000 & 29.7 & 43.9 & 395. & 2.08 & $\mathbf{1 . 1 8}$ & 1.55 \\
ecl32 & Fail & Fail & 562. & Fail & 145. & $\mathbf{4 1 . 2}$ \\
epb3 & 29.7 & 24.2 & 5.00 & 5.67 & 5.69 & $\mathbf{2 . 1 6}$ \\
fidap011 & 168. & 39.9 & 12.2 & 16.9 & 18.7 & $\mathbf{6 . 3 8}$ \\
fidapm11 & 944. & 88.9 & 15.1 & Fail & 25.3 & $\mathbf{1 1 . 2}$ \\
lhr71c & 6.80 & 12.5 & Fail & 23.0 & 11.7 & $\mathbf{3 . 0 5}$ \\
nasasrb & 81.8 & 102. & 25.0 & Fail & 26.8 & $\mathbf{1 0 . 9}$ \\
onetone1 & 12.2 & 184. & 113. & 10.7 & 10.0 & $\mathbf{7 . 2 5}$ \\
onetone2 & 1.79 & 28.3 & 20.7 & 3.55 & 2.81 & $\mathbf{1 . 1 1}$ \\
pre2 & Fail & Fail & Fail & Fail & Fail & $\mathbf{3 6 2 .}$ \\
raefsky3 & 39.0 & 146. & 10.0 & 6.86 & 8.75 & $\mathbf{4 . 5 4}$ \\
raefsky4 & 109. & 1983 & 157. & 28.1 & 27.5 & $\mathbf{7 . 7 8}$ \\
rma10 & 15.7 & Fail & 10.7 & 5.78 & 9.62 & $\mathbf{3 . 7 6}$ \\
tib & 0.52 & 266. & 1.75 & 1.47 & 0.62 & $\mathbf{0 . 3 1}$ \\
twotone & $\mathbf{3 0 . 0}$ & Fail & 724. & 637. & 124. & 37.9 \\
venkat50 & 16.2 & Fail & 11.6 & 8.11 & 19.4 & $\mathbf{4 . 4 0}$ \\
wang3 & 106. & 3226 & 62.7 & 36.9 & 32.3 & $\mathbf{1 3 . 4}$ \\
wang4 & 97.3 & 318. & 16.2 & 23.7 & 25.6 & $\mathbf{1 2 . 0}$ \\
\hline \hline
\end{tabular}

- Task dependency graph: Tree.

- Numerical factorization: Supernodal Crout.

- Pivoting strategy: Threshold pivoting implemented by row-exchanges.

\section{SPOOLES 4]}

- Fill reducing ordering: Generalized nested dissection/multisection [5] on the symmetric structure of $A+A^{T}$.

- Task dependency graph: Tree.

- Numerical factorization: Supernodal Crout.

- Pivoting strategy: Threshold rook pivoting that may perform both row and column exchanges to control growth in both $L$ and $U$.

4. SuperLU $U_{\text {dist } 2223]}$

- Fill reducing ordering: Multiple minimum degree [13] on the symmetric structure of $A+A^{T}$.

- Task dependency graph: Directed acyclic graph.

- Numerical factorization: Supernodal right-looking. 
- Pivoting strategy: No numerical pivoting during factorization. Rows are preordered to maximize the magnitude of the product of the diagonal entries [1].

\section{MUMPS [12]}

- Fill reducing ordering: Approximate minimum degree [7] on the symmetric structure of $A+A^{T}$.

- Task dependency graph: Tree.

- Numerical factorization: Symmetric-pattern multifrontal.

- Pivoting strategy: Preordering rows to maximize the magnitude of the product of the diagonal entries [11], followed by unsymmetric row exchanges within supernodes and symmetric row and column exchanges between supernodes.

\section{WSMP [1517]}

- Fill reducing ordering: Nested dissection [1816] on the symmetric structure of $A+A^{T}$.

- Task dependency graph: Minimal directed acyclic graph [15].

- Numerical factorization: Unsymmetric-pattern multifrontal.

- Pivoting strategy: Preordering rows to maximize the magnitude of the product of the diagonal entries [11], followed by unsymmetric partial pivoting within supernodes and symmetric pivoting between supernodes. Rook pivoting (which attempts to contain growth in both $L$ and $U$ ) is an option.

The multifrontal method 1224 for the solving sparse systems of linear equations offers a significant performance advantage over more conventional factorization schemes by permitting efficient utilization of parallelism and memory hierarchy. Our detailed experiments in 19 show that all three multifrontal solvers-UMFPACK, MUMPS, and WSMP - run at a much higher Megaflop rate than their non-multifrontal counterparts. The original multifrontal algorithm proposed by Duff and Reid [12] uses the symmetric pattern of $A+A^{T}$ to generate an elimination tree to guide the numerical factorization, which works on symmetric frontal matrices. This symmetric-pattern multifrontal algorithm used in MUMPS can incur a substantial overhead for very unsymmetric matrices due to unnecessary dependencies in the elimination tree and extra zeros in the artificially symmetric frontal matrices. Davis and Duff 8 and Hadfield 21] introduced an unsymmetric-pattern multifrontal algorithm, which is used in UMFPACK and overcomes the shortcomings of the symmetric-pattern multifrontal algorithm. However, UMFPACK did not reveal the full potential of the unsymmetric-pattern multifrontal algorithm because of the choice of a fill-reducing ordering (AMD), which has now been shown to be less effective than nested dissection [3]. Moreover, the merging of the ordering and symbolic factorization within numerical factorization slowed down the latter and excluded the possibility of using a better ordering while retaining the factorization code. Other than WSMP, SPOOLES is the only solver that uses a graph-partioning based ordering. However, it appears that the fill-in resulting from rook pivoting, 
which involves both row and column exchanges in an attempt to limit pivot growth in both $L$ and $U$, overshadows a good initial ordering. Simple threshold partial pivoting yields a sufficiently accurate factorization for most matrices, including all our test cases. Therefore, rook pivoting is an option in WSMP, but the default is a simple threshold pivoting.

WSMP achieves superior levels of performance by incorporating the best ideas of the previous solvers into one package, while avoiding their pitfalls and by introducing new techniques in both symbolic and numerical phases. The analysis or preprocessing phase of WSMP uses a reduction to a block-triangular form [10], a permutation of rows to maximize the magnitude of the product of the diagonal entries [1120, a multilevel nested dissection ordering [16] for fill-in reduction, and an improved version [15] of the classical unsymmetric symbolic factorization algorithm [14 to determine the supernodal structure of factors and the minimal directed acyclic task- and data-dependency graphs to guide potentially multiple steps of numerical factorization with minimum overhead and maximum parallelism. The unsymmetric-pattern multifrontal LU factorization algorithm of WSMP [15] uses novel data-structures to efficiently handle any amount of pivoting and different pivot sequences without repeating the symbolic phase for each factorization.

\section{Performance on a Shared-Memory Parallel Workstation}

Having empirically established in Section 2 that MUMPS and WSMP are the fastest and the most robust among the currently available general sparse solvers, in Table 3 we give 1- and 4-CPU factorization times of these two solvers on an IBM RS6000 workstation with $375 \mathrm{Mhz}$ Power 3 processors. These processors have a peak theoretical speed of 1.5 Gigaflops. They share a $4 \mathrm{MB}$ level-2 cache and have a $64 \mathrm{~KB}$ level-1 cache each. 2 GB of memory was available to each single CPU run and the 4-CPU runs of WSMP. MUMPS, when run on 4 processors, had a total of $4 \mathrm{~GB}$ of memory available to it.

The relative performance of WSMP improves on the Power 3 as it is able to extract a higher Megaflop rate from this machine. The most noteworthy observation from Table 3 (last column) is that out of the 25 test cases, only 3 require more than 10 seconds on a mere workstation and all but one of the matrices can be factored in under 13 seconds. The factorization times reported in Table 3 use the default options of WSMP. Many of the large test matrices, such as fidapm11, mil053, mixtank, nasasrb, raefsky3, raefsky4, rma10, venkat50, wang3, and wang 4 have a symmetric structure and would need even less factorization time if the user switches off the optional pre-permutation of rows to maximize the diagonal magnitudes. This row permutation, which is on by default in WSMP, destroys the structural symmetry and increases the fill-in and operation count of factorization. Some of the matrices, such as mil053, venkat50, wang3, wang4, etc. do not require partial pivoting to yield an accurate factorization. Therefore, if the user familiar with the characteristics of the matrices, switches off the piv- 
Table 3. Number of factor nonzeros, operation count, and LU factorization (with partial pivoting) times of MUMPS and WSMP on one and four $375 \mathrm{Mhz}$ Power 3 processors.

\begin{tabular}{||l||c|c||c|c||c|c||c|c||}
\hline \hline \multirow{2}{*}{ Matrix } & \multicolumn{5}{c|}{ MUMPS } & \multicolumn{4}{c||}{ WSMP } \\
\cline { 2 - 4 } & $\begin{array}{c}\text { NNZ }_{L U} \\
\times 10^{6}\end{array}$ & $\begin{array}{c}\text { Ops } \\
\times 10^{9}\end{array}$ & $\begin{array}{c}1 \\
\text { CPU }\end{array}$ & $\begin{array}{c}4 \\
\text { CPUs }\end{array}$ & $\begin{array}{c}\text { NNZ } \\
\times 10^{6}\end{array}$ & $\begin{array}{c}\text { Ops } \\
\times 10^{9}\end{array}$ & $\begin{array}{c}1 \\
\text { CPU }\end{array}$ & $\begin{array}{c}4 \\
\text { CPUs }\end{array}$ \\
\hline \hline af23560 & 8.34 & 2.56 & $\mathbf{7 . 7 5}$ & $\mathbf{3 . 8 2}$ & 11.1 & 3.27 & $\mathbf{4 . 1 7}$ & $\mathbf{2 . 1 3}$ \\
\hline av41092 & 14.1 & 8.42 & $\mathbf{1 9 . 8}$ & $\mathbf{1 0 . 7}$ & 9.28 & 2.02 & $\mathbf{4 . 7 1}$ & $\mathbf{2 . 9 0}$ \\
\hline bayer01 & 2.82 & .125 & $\mathbf{2 . 2 3}$ & $\mathbf{1 . 2 6}$ & 1.75 & .040 & $\mathbf{1 . 3 4}$ & $\mathbf{1 . 3 3}$ \\
\hline bbmat & 46.0 & 41.4 & $\mathbf{7 6 . 3}$ & $\mathbf{3 2 . 2}$ & 36.6 & 20.1 & $\mathbf{2 3 . 7}$ & $\mathbf{8 . 6 8}$ \\
\hline comp2c & 7.05 & 4.22 & $\mathbf{2 2 . 3}$ & $\mathbf{1 3 . 6}$ & 3.53 & 1.09 & $\mathbf{2 . 7 2}$ & $\mathbf{1 . 0 2}$ \\
\hline e40r0000 & 1.72 & .172 & $\mathbf{1 . 7 0}$ & $\mathbf{1 . 2 0}$ & 2.32 & .250 & $\mathbf{0 . 6 2}$ & $\mathbf{0 . 3 7}$ \\
\hline ecl32 & 42.9 & 64.6 & $\mathbf{8 2 . 6}$ & $\mathbf{4 1 . 0}$ & 30.3 & 21.0 & $\mathbf{2 3 . 9}$ & $\mathbf{8 . 7 1}$ \\
\hline epb3 & 6.90 & 1.17 & $\mathbf{5 . 1 0}$ & $\mathbf{2 . 4 9}$ & 5.64 & .451 & $\mathbf{1 . 9 4}$ & $\mathbf{1 . 9 0}$ \\
\hline fidap011 & 12.5 & 7.01 & $\mathbf{1 4 . 5}$ & $\mathbf{1 1 . 8}$ & 10.2 & 3.20 & $\mathbf{4 . 2 2}$ & $\mathbf{2 . 0 1}$ \\
\hline fidapm11 & 14.0 & 9.67 & $\mathbf{1 8 . 2}$ & $\mathbf{1 0 . 2}$ & 14.9 & 5.21 & $\mathbf{6 . 7 7}$ & $\mathbf{2 . 7 3}$ \\
\hline invextr1 & 30.3 & 35.6 & $\mathbf{5 3 . 6}$ & $\mathbf{2 9 . 8}$ & 16.3 & 6.90 & $\mathbf{1 0 . 8}$ & $\mathbf{6 . 3 6}$ \\
\hline lhr34c & 5.58 & .641 & $\mathbf{5 . 3 0}$ & $\mathbf{2 . 4 3}$ & 3.47 & .170 & $\mathbf{1 . 1 6}$ & $\mathbf{1 . 1 3}$ \\
\hline mil053 & 75.9 & 31.8 & $\mathbf{6 8 . 8}$ & $\mathbf{2 6 . 0}$ & 66.2 & 14.4 & $\mathbf{2 4 . 8}$ & $\mathbf{1 2 . 9}$ \\
\hline mixtank & 38.5 & 64.4 & $\mathbf{8 0 . 0}$ & $\mathbf{3 8 . 4}$ & 27.0 & 19.5 & $\mathbf{2 2 . 9}$ & $\mathbf{9 . 3 4}$ \\
\hline nasasrb & 24.2 & 9.45 & $\mathbf{2 3 . 0}$ & $\mathbf{1 6 . 4}$ & 21.7 & 5.41 & $\mathbf{7 . 2 2}$ & $\mathbf{3 . 8 6}$ \\
\hline onetone1 & 4.72 & 2.29 & $\mathbf{7 . 0 6}$ & $\mathbf{4 . 2 1}$ & 4.34 & 1.79 & $\mathbf{3 . 8 8}$ & $\mathbf{1 . 9 6}$ \\
\hline onetone2 & 2.26 & .510 & $\mathbf{2 . 3 3}$ & $\mathbf{1 . 4 1}$ & 1.62 & .206 & $\mathbf{0 . 9 0}$ & $\mathbf{0 . 9 4}$ \\
\hline pre2 & 358. & Fail & Fail & Fail & 97.5 & 133. & $\mathbf{1 8 9}$ & $\mathbf{7 7 . 0}$ \\
\hline raefsky3 & 8.44 & 2.90 & $\mathbf{8 . 3 7}$ & $\mathbf{5 . 5 8}$ & 9.42 & 2.57 & $\mathbf{3 . 2 7}$ & $\mathbf{1 . 5 4}$ \\
\hline raefsky4 & 15.7 & 10.9 & $\mathbf{1 9 . 9}$ & $\mathbf{1 3 . 2}$ & 11.8 & 4.11 & $\mathbf{5 . 0 9}$ & $\mathbf{2 . 5 4}$ \\
\hline rma10 & 8.87 & 1.40 & $\mathbf{8 . 4 1}$ & $\mathbf{5 . 3 8}$ & 10.9 & 1.48 & $\mathbf{2 . 5 9}$ & $\mathbf{1 . 0 2}$ \\
\hline twotone & 22.1 & 29.3 & $\mathbf{6 8 . 7}$ & $\mathbf{3 2 . 1}$ & 12.5 & 10.4 & $\mathbf{1 8 . 3}$ & $\mathbf{1 2 . 9}$ \\
\hline venkat50 & 12.0 & 2.31 & $\mathbf{9 . 7 5}$ & $\mathbf{5 . 2 7}$ & 12.9 & 1.75 & $\mathbf{3 . 0 1}$ & $\mathbf{1 . 2 4}$ \\
\hline wang3 & 13.8 & 13.8 & $\mathbf{2 0 . 1}$ & $\mathbf{8 . 3 4}$ & 11.9 & 5.91 & $\mathbf{6 . 9 2}$ & $\mathbf{3 . 3 1}$ \\
\hline wang4 & 11.6 & 10.5 & $\mathbf{1 6 . 6}$ & $\mathbf{7 . 6 7}$ & 12.2 & 6.09 & $\mathbf{7 . 0 6}$ & $\mathbf{3 . 5 1}$ \\
\hline \hline & & & & & & & & \\
\hline
\end{tabular}

oting for these matrices and, in general, tailors the various options [17] to her application, many of the test problems can be solved even faster.

\section{Concluding Remarks}

In this paper, we show that recent sparse solvers have improved the state of the art of the direct solution of general sparse systems by almost an order of magnitude. Coupled with the good scalability of these solvers [3] and the availability of relatively inexpensive high-performance parallel computers, it is now possible to solve very large sparse systems in only a small fraction of the time that these solutions would have required just a few years ago. Judging by the availability of real test cases, it appears that the applications that require the solution of such 
systems have not kept pace with the improvements in the software and hardware available to solve these systems. We hope that the new sparse solvers will encourage scientists and engineers to develop bigger models with larger sparse systems so that the full potential of the new generation of parallel sparse general solvers can be exploited.

\section{References}

1. Patrick R. Amestoy, Iain S. Duff, Jacko Koster, and J. Y. L'Execellent. A fully asynchronous multifrontal solver using distributed dynamic scheduling. Technical Report RT/APO/99/2, ENSEEIHT-IRIT (Toulouse, France), 1999. To appear in SIAM Journal on Matrix Analysis and Applications.

2. Patrick R. Amestoy, Iain S. Duff, and J. Y. L'Execellent. Multifrontal parallel distributed symmetric and unsymmetric solvers. Computational Methods in Applied Mechanical Engineering, 184:501-520, 2000. Also available at http://www.enseeiht.fr/apo/MUMPS/.

3. Patrick R. Amestoy, Iain S. Duff, J. Y. L'Execellent, and Xiaoye S. Li. Analysis, tuning, and comparison of two general sparse solvers for distributed memory computers. Technical Report RT/APO/00/2, ENSEEIHT-IRIT, Toulouse, France, 2000. Also available as Technical Report 45992 from Lawrence Berkeley National Laboratory.

4. Cleve Ashcraft and Roger G. Grimes. SPOOLES: An object-oriented sparse matrix library. In Proceedings of the Ninth SIAM Conference on Parallel Processing for Scientific Computing, March 1999.

5. Cleve Ashcraft and Joseph W.-H. Liu. Robust ordering of sparse matrices using multisection. Technical Report CS 96-01, Department of Computer Science, York University, Ontario, Canada, 1996.

6. Michel Cosnard and Laura Grigori. Using postordering and static symbolic factorization for parallel sparse LU. In Proceedings of the International Parallel and Distributed Processing Symposium (IPDPS), 2000.

7. Timothy A. Davis, Patrick Amestoy, and Iain S. Duff. An approximate minimum degree ordering algorithm. Technical Report TR-94-039, Computer and Information Sciences Department, University of Florida, Gainesville, FL, 1994.

8. Timothy A. Davis and Iain S. Duff. An unsymmetric-pattern multifrontal method for sparse LU factorization. SIAM Journal on Matrix Analysis and Applications, 18(1):140-158, January 1997.

9. James W. Demmel, Stanley C. Eisenstat, John R. Gilbert, Xiaoye S. Li, and Joseph W.-H. Liu. A supernodal approach to sparse partial pivoting. SIAM Journal on Matrix Analysis and Applications, 20(3):720-755, 1999.

10. Iain S. Duff, A. M. Erisman, and John K. Reid. Direct Methods for Sparse Matrices. Oxford University Press, Oxford, UK, 1990.

11. Iain S. Duff and Jacko Koster. On algorithms for permuting large entries to the diagonal of a sparse matrix. Technical Report RAL-TR-1999-030, Rutherford Appleton Laboratory, April 19, 1999.

12. Iain S. Duff and John K. Reid. The multifrontal solution of unsymmetric sets of linear equations. SIAM Journal on Scientific and Statistical Computing, 5(3):633641, 1984.

13. Alan George and Joseph W.-H. Liu. Computer Solution of Large Sparse Positive Definite Systems. Prentice-Hall, NJ, 1981. 
14. John R. Gilbert and Joseph W.-H. Liu. Elimination structures for unsymmetric sparse LU factors. SIAM Journal on Matrix Analysis and Applications, 14(2):334352, 1993.

15. Anshul Gupta. A high-performance GEPP-based sparse solver. Technical report, IBM T. J. Watson Research Center, Yorktown Heights, NY, 2001. ftp://ftp.cs.umn.edu/users/kumar/anshul/parco-01.ps.

16. Anshul Gupta. Fast and effective algorithms for graph partitioning and sparse matrix ordering. IBM Journal of Research and Development, 41(1/2):171-183, January/March, 1997.

17. Anshul Gupta. WSMP: Watson sparse matrix package (Part-II: direct solution of general sparse systems). Technical Report RC 21888 (98472), IBM T. J. Watson Research Center, Yorktown Heights, NY, November 20, 2000.

http://www.cs.umn.edu/〜agupta/wsmp.html.

18. Anshul Gupta. Graph partitioning based sparse matrix ordering algorithms for finite-element and optimization problems. In Proceedings of the Second SIAM Conference on Sparse Matrices, October 1996.

19. Anshul Gupta and Yanto Muliadi. An experimental comparison of some direct sparse solver packages. Technical Report RC 21862 (98393), IBM T. J. Watson Research Center, Yorktown Heights, NY, October 25, 2000. ftp://ftp.cs.umn.edu/users/kumar/anshul/solver-compare.ps.

20. Anshul Gupta and Lexing Ying. Algorithms for finding maximum matchings in bipartite graphs. Technical Report RC 21576 (97320), IBM T. J. Watson Research Center, Yorktown Heights, NY, October 19, 1999.

21. Steven Michael Hadfield. On the LU Factorization of Sequences of Identically Structured Sparse Matrices within a Distributed Memory Environment. PhD thesis, University of Florida, Gainsville, FL, 1994.

22. Xiaoye S. Li and James W. Demmel. Making sparse Gaussian elimination scalable by static pivoting. In Supercomputing '98 Proceedings, 1998.

23. Xiaoye S. Li and James W. Demmel. A scalable sparse direct solver using static pivoting. In Proceedings of the Ninth SIAM Conference on Parallel Processing for Scientific Computing, 1999.

24. Joseph W.-H. Liu. The multifrontal method for sparse matrix solution: Theory and practice. SIAM Review, 34:82-109, 1992.

25. Olaf Schenk, Wolfgang Fichtner, and Klaus Gartner. Scalable parallel sparse LU factorization with a dynamical supernode pivoting approach in semiconductor device simulation. Technical Report 2000/10, Integrated Systems Laboratory, Swiss Federal Institute of Technology, Zurich, November 2000.

26. Kai Shen, Tao Yang, and Xiangmin Jiao. S+: Efficient 2D sparse LU factorization on parallel machines. SIAM Journal on Matrix Analysis and Applications, To be published. 